Algebraic $8 \mathcal{G}$ Geometric $\mathcal{T}$ opology

Volume 5 (2005) 53-69

Published: 9 January 2005

ATG

\title{
On some anticyclic operads
}

\author{
F. CHApoton
}

\begin{abstract}
Some binary quadratic operads are endowed with anticyclic structures and their characteristic functions as anticyclic operads are determined, or conjectured in one case.
\end{abstract}

AMS Classification 18D50; 05E05

Keywords Anticyclic operad, Legendre transform

\section{Introduction}

The most classical operads are probably the three operads describing commutative and associative algebras, associative algebras and Lie algebras. They fit in the following diagram:

$$
\text { Comm } \longleftarrow \text { Assoc } \longleftarrow \text { Lie }
$$

These operads have very nice properties. First of all, they are binary quadratic and Koszul in the sense of the work of Ginzburg and Kapranov on the Koszul duality of operads [7]. More precisely Assoc is self-dual and Lie and Comm are dual to each other. Moreover they are basic examples of cyclic operads, a notion introduced in [5] and related to the moduli spaces of curves with marked points. The developments of Koszul duality of operads and of the theory of cyclic operads were both partly motivated by the work of Kontsevich on noncommutative symplectic geometry [9], where three parallel constructions are made for these three operads.

Our aim is to explain that most of the properties of this classical sequence of operads also hold for two other diagrams involving some binary quadratic operads introduced by Loday [1] and others [2, 3]. The first of these diagrams is

$$
\text { Perm } \longleftarrow \text { Dias } \longleftarrow \text { Leib }
$$

and the other one is:

$$
\text { Zinb } \longleftarrow \text { Dend } \longleftarrow \text { PreLie }
$$


All these operads are already known to be binary quadratic and Koszul. More precisely, (Perm, PreLie), (Dias, Dend) and (Leib, Zinb) are Koszul dual pairs of operads. The main objective of this article is to show that they are anticyclic operads and that the maps in the two diagrams above are maps of anticyclic operads.

One interesting point with cyclic and anticyclic operads is that they allow the building of Lie algebras and graph complexes 6, 4, 12. There has been a lot of work on the graph complexes for the three classical cyclic operads Comm, Assoc and Lie. It may be worth considering the analogous structures for the six new anticyclic operads introduced here.

Let us also remark that there is a fourth classical cyclic operad, namely the Poisson operad, which can be obtained as the graded cyclic operad associated to a filtration of the cyclic operad Assoc. Similar objects exists in the diagrams above, namely filtrations of the anticyclic operads Dias and Dend and associated graded anticyclic operads, one of which is related to the pre-Poisson algebras studied in [1].

\section{Anticyclic operads}

We briefly state some general facts on operads and anticyclic operads. A convenient reference on this subject is [12, see also [7, [5]. Most of the operads considered here will be in the monoidal category of vector spaces over the field $\mathbb{Q}$, but the true ambient category is the category of chain complexes of vector spaces over $\mathbb{Q}$.

Recall that an operad $\mathcal{P}$ is a collection of modules $\mathcal{P}(n)$ over the symmetric groups $\mathfrak{S}_{n}$ together with composition maps satisfying some axioms modelled after the composition of multi-linear maps. A non-symmetric operad $\mathcal{P}$ is a similar structure without the actions of the symmetric groups. If $\mathcal{P}$ is a nonsymmetric operad then the collection $\mathcal{P}(n) \otimes \mathbb{Q S}_{n}$ is naturally an operad.

An anticyclic non-symmetric operad is a non-symmetric operad $\mathcal{P}$ together with an action of a cyclic group of order $n+1$ on $\mathcal{P}(n)$ satisfying some axioms. In particular, the action of the cyclic group is determined by the action on the generators of the non-symmetric operad.

Similarly, an anticyclic operad is an operad $\mathcal{P}$ together with an action of a symmetric group $\mathfrak{S}_{n+1}$ on $\mathcal{P}(n)$ extending the action of $\mathfrak{S}_{n}$ and satisfying

similar axioms. Here the group $\mathfrak{S}_{n}$ is embedded in $\mathfrak{S}_{n+1}$ as the stabilizer of 
$n+1$. In this case too, the action of the big symmetric group is determined by the action on the generators of the operad.

The main difference with the notion of cyclic operad is that the unit 1 of the operad is mapped to -1 in an anticyclic case and to 1 in the cyclic case. There is also some change of sign in the axioms.

The Hadamard product of two operads $\mathcal{P}_{1}$ and $\mathcal{P}_{2}$ is an operad with underlying modules $\mathcal{P}_{1}(n) \otimes \mathcal{P}_{2}(n)$. If $\mathcal{P}_{1}$ and $\mathcal{P}_{2}$ are cyclic or anticyclic operads, then the Hadamard product is an anticyclic or cyclic operad for the tensor product action of the symmetric groups $\mathfrak{S}_{n+1}$. Whether the Hadamard product is cyclic or anticyclic is determined by the action on the unit 1, i.e. is given by a sign rule where "cyclic" is +1 and "anticyclic" is -1 .

Let $\mathbb{Z} /(n+1) \mathbb{Z}$ be the subgroup of $\mathfrak{S}_{n+1}$ generated by the longest cycle $(n+$ $1, n, \ldots, 2,1)$. If $\mathcal{P}$ is an anticyclic non-symmetric operad, then the collection of induced modules $\operatorname{Ind}_{\mathbb{Z} /(n+1) \mathbb{Z}}^{\mathfrak{S}_{n+1}} \mathcal{P}(n)$ has a natural structure of anticyclic operad.

There exists a differential graded anticyclic operad, called the determinant operad and denoted by Det, such that $\operatorname{Det}(n)$ is a chain complex of dimension 1 concentrated in degree $n-1$. The Hadamard product by the operad Det corresponds to the suspension of operads. It maps anticyclic operads to cyclic operads and vice-versa.

\section{Koszul duality and Legendre transform}

The theory of Koszul duality for operads has been introduced in [7 for binary quadratic operads.

For each binary quadratic operad $\mathcal{P}$, one can define its dual operad $\mathcal{P}^{!}$by elementary linear algebra using the quadratic presentation of $\mathcal{P}$. In particular, the space $\mathcal{P}^{!}(2)$ of generators of $\mathcal{P}^{!}$is the tensor product of the dual space of $\mathcal{P}(2)$ by the sign representation of $\mathfrak{S}_{2}$.

A binary quadratic operad $\mathcal{P}$ is Koszul if the natural morphisms of cooperads from the bar complex of $\mathcal{P}$ to the dual cooperad of the suspension of $\mathcal{P}^{!}$is a quasi-isomorphism.

The Koszul dual operad $\mathcal{P}^{!}$of a Koszul operad $\mathcal{P}$ has a natural structure of cyclic (resp. anticyclic) operad if $\mathcal{P}$ is anticyclic (resp. anticyclic). When $\mathcal{P}$ is cyclic or anticyclic, the action of the cycle $(321)$ on $\mathcal{P}^{!}(2)$ is given by the transpose of the inverse of the action on the space $\mathcal{P}(2)$. 
When $\mathcal{P}$ is cyclic (resp. anticyclic), both the bar complex and the dual cooperad of the suspension of $\mathcal{P}^{!}$are anticyclic (resp. cyclic) and the natural map between them is automatically a morphism of anticyclic (resp. cyclic) cooperads.

It is known that generating series of dual Koszul operads are related by inversion for the plethysm. More precisely, let the characteristic function of an operad $\mathcal{P}$ be

$$
\operatorname{ch}(\mathcal{P})=\sum_{n \geq 1} \operatorname{ch}_{n}(\mathcal{P}(n))
$$

where $\operatorname{ch}_{n}(\mathcal{P}(n))$ is the symmetric function for the $\mathfrak{S}_{n}$-module $\mathcal{P}(n)$. The suspension $\Sigma F$ of a symmetric function $F$ is defined as follows:

$$
\Sigma F=-F\left(-p_{1},-p_{2},-p_{3}, \ldots\right),
$$

where the $p_{k}$ are power sums symmetric functions. Then one has the following relation for a Koszul operad $\mathcal{P}$ :

$$
\operatorname{ch}(\mathcal{P}) \circ \Sigma \operatorname{ch}\left(\mathcal{P}^{!}\right)=p_{1},
$$

where $\circ$ is the plethysm of symmetric functions.

A similar relation exists at the level of cyclic or anticyclic Koszul operads, where the generating functions are related by the Legendre transform of symmetric functions introduced in [5]. Let us give the precise statement.

The characteristic function of a cyclic or anticyclic operad $\mathcal{P}$ is defined by

$$
\mathbf{C h}(\mathcal{P})=\sum_{n \geq 1} \mathbf{C h}_{n+1}(\mathcal{P}(n)),
$$

where $\mathbf{C h}_{n+1}(\mathcal{P}(n))$ is the symmetric function for the $\mathfrak{S}_{n+1}$-module $\mathcal{P}(n)$.

Let $F$ be a symmetric function with no term of degree 0 and 1 and such that the term of degree 1 of $\partial_{p_{1}} F$ does not vanish. The Legendre transform $G=\mathcal{L} F$ of $F$ is defined by

$$
F \circ \frac{\partial G}{\partial p_{1}}+G=p_{1} \frac{\partial G}{\partial p_{1}}
$$

The Legendre transform is an involution, with the property that the derived symmetric functions satisfy

$$
\frac{\partial F}{\partial p_{1}} \circ \frac{\partial G}{\partial p_{1}}=p_{1}
$$

Then if $\mathcal{P}$ is an anticyclic (resp. cyclic) operad which is Koszul as an operad, one has

$$
\mathcal{L} \mathbf{C h}(\mathcal{P})=-\Sigma \mathbf{C h}\left(\mathcal{P}^{!}\right),
$$

where the anticyclic (resp. cyclic) structure on $\mathcal{P}^{!}$is the one induced by the Koszul duality. 


\section{The diassociative operad}

In this section and the next one, we will consider two non-symmetric operads.

First let us recall some known facts about the non-symmetric operad Dias of diassociative algebras, see [11].

First, it has a quadratic presentation. The generators are two binary operations $x \dashv y$ and $x \vdash y$. These generators satisfy the following relations:

$$
\begin{aligned}
x \dashv(y \vdash z)= & x \dashv(y \dashv z)=(x \dashv y) \dashv z, \\
& x \vdash(y \dashv z)=(x \vdash y) \dashv z, \\
x \vdash(y \vdash z)= & (x \vdash y) \vdash z=(x \dashv y) \vdash z .
\end{aligned}
$$

Next, it is known that the space $\operatorname{Dias}(n)$ has dimension $n$ with a base $\left(e_{m}^{n}\right)_{m=1, \ldots, n}$ such that the composition in the operad is given by

$$
e_{m}^{n} \circ_{i} e_{k}^{\ell}= \begin{cases}e_{m+\ell-1}^{n+\ell-1} & \text { if } i=m, \\ e_{m+\ell-1}^{n+\ell-1} & \text { if } i<m, \\ e_{m}^{n+\ell-1} & \text { if } i>m .\end{cases}
$$

In the presentation above, $x_{1} \dashv x_{2}$ is $e_{1}^{2}$ and $x_{1} \vdash x_{2}$ is $e_{2}^{2}$. More generally, the element $e_{m}^{n}$ is mapped to

$$
x_{1} \vdash x_{2} \vdash \cdots \vdash x_{m} \dashv \cdots \dashv x_{n},
$$

with arbitrary parentheses. Conversely, any iterated product of the variables $x_{1}, x_{2}, \ldots, x_{n}$ in this order corresponds to some $e_{m}^{n}$ by the following recursive procedure. At each step, choose the sub-expression which is not on the bar side of $\dashv$ or $\vdash$, until there remains only one variable $x_{m}$. For example,

$$
\left(\left(x_{1} \dashv x_{2}\right) \vdash x_{3}\right) \dashv\left(x_{4} \vdash x_{5}\right)
$$

is mapped to $e_{3}^{5}$.

Let us now introduce a notion of invariant bilinear map on a diassociative algebra. It is an antisymmetric map with value in some vector space:

$$
\langle x, y\rangle=-\langle y, x\rangle,
$$

such that

$$
\langle x \dashv y, z\rangle=-\langle y \vdash z, x\rangle \text { and }\langle x \vdash y, z\rangle=\langle y \dashv z, x\rangle-\langle y \vdash z, x\rangle .
$$

Let us define a map $\tau_{1}$ as multiplication by -1 on $\operatorname{Dias}(1)$ and a map $\tau_{2}$ on $\operatorname{Dias}(2)$ by the following relation

$$
\langle E(x, y), z\rangle=\left\langle\tau_{2}(E)(y, z), x\right\rangle
$$

for each element $E$ in $\left\{e_{1}^{2}, e_{2}^{2}\right\}$, using the previous conditions on $\langle$,$\rangle .$ 
Theorem 3.1 There exists a unique collection $\tau$ of endomorphisms $\tau_{n}$ of the space Dias $(n)$ extending $\tau_{1}$ and $\tau_{2}$ and endowing the operad Dias with a structure of non-symmetric anticyclic operad.

Proof Clearly $\tau_{1}$ is of order 2 and $\tau_{2}$ is of order 3. As Dias is generated by $\operatorname{Dias}(2)$, the structure map $\tau$ is unique if it exists. To check that $\tau_{n}$ can be defined for $n \geq 3$, it is enough to check that the notion of invariant form is compatible with the relations defining the operad Dias. Let us check one of these compatibility conditions, for the relation (12):

$$
\begin{gathered}
\langle x \vdash(y \dashv z)-(x \vdash y) \dashv z, t\rangle \\
=\langle(y \dashv z) \dashv t-(y \dashv z) \vdash t, x\rangle+\langle z \vdash t, x \vdash y\rangle \\
=\langle(y \dashv z) \dashv t-(y \dashv z) \vdash t, x\rangle-\langle x \vdash y, z \vdash t\rangle \\
=\langle(y \dashv z) \dashv t-(y \dashv z) \vdash t, x\rangle-\langle y \dashv(z \vdash t)-y \vdash(z \vdash t), x\rangle=0,
\end{gathered}
$$

where one has used the antisymmetry and the invariance to obtain an expression with the $x$ variable only in the right slot. The remaining checks are quite similar and are left to the reader.

In fact, $\tau_{n}$ has a very simple expression in the base $e^{n}$.

Lemma 3.2 The action of $\tau_{n}$ is given by

$$
\tau_{n}\left(e_{m}^{n}\right)= \begin{cases}-e_{n}^{n} & \text { if } m=1 \\ -e_{n}^{n}+e_{m-1}^{n} & \text { else. }\end{cases}
$$

Proof It is readily true for $n=1$ and 2 . Let us first prove that $\tau_{n+1}\left(e_{1}^{n+1}\right)=$ $e_{n+1}^{n+1}$. This follows from the equality:

$$
\begin{aligned}
\left\langle x_{1} \dashv x_{2} \dashv \cdots \dashv x_{n+1}, x_{n+2}\right\rangle & =-\left\langle\left(x_{2} \dashv \cdots \dashv x_{n+1}\right) \vdash x_{n+2}, x_{1}\right\rangle \\
& =-\left\langle x_{2} \vdash \cdots \vdash x_{n+2}, x_{1}\right\rangle .
\end{aligned}
$$

Let us now compute $\tau_{n+1}\left(e_{m}^{n+1}\right)$ for $m \geq 2$. Indeed, one has

$$
\begin{gathered}
\left\langle x_{1} \vdash x_{2} \vdash \cdots \vdash x_{m} \dashv \cdots \dashv x_{n+1}, x_{n+2}\right\rangle= \\
\left\langle\left(x_{2} \vdash \cdots \vdash x_{m} \dashv \cdots \dashv x_{n+1}\right) \dashv x_{n+2}-\left(x_{2} \vdash \cdots \vdash x_{m} \dashv \cdots \dashv x_{n+1}\right) \vdash x_{n+2}, x_{1}\right\rangle \\
=\left\langle x_{2} \vdash \cdots \vdash x_{m} \dashv \cdots \dashv x_{n+2}-x_{2} \vdash \cdots \vdash x_{n+2}, x_{1}\right\rangle .
\end{gathered}
$$

This concludes the proof.

One can note that the matrix of $\tau_{n}$ in the base $e^{n}$ is a companion matrix for the polynomial $1+x+\cdots+x^{n}$ and can also be described as $-\left({ }^{t} L\right)^{-1} L$ where $L$ is the lower triangular matrix with 1 everywhere below the diagonal. 


\section{The dendriform operad}

Let us now recall some facts about the non-symmetric operad Dend describing dendriform algebras, see [1].

First, it also has a quadratic presentation. The generators are two binary operations $x \prec y$ and $x \succ y$. These generators satisfy the following relations:

$$
\begin{aligned}
&(x \prec y) \prec z)= x \prec(y \prec z)+x \prec(y \succ z), \\
& x \succ(y \prec z)=(x \succ y) \prec z, \\
& x \succ(y \succ z)=(x \succ y) \succ z+(x \prec y) \succ z .
\end{aligned}
$$

Next, it is known that the dimension of the space $\operatorname{Dend}(n)$ is the Catalan number $c_{n}=\frac{1}{n+1}\left(\begin{array}{c}2 n \\ n\end{array}\right)$. There is a base $Y^{n}$ of $\operatorname{Dend}(n)$ indexed by planar binary trees with $n+1$ leaves, in which the composition of the operad has a simple expression. The base $Y^{2}$ is made precisely of $x_{1} \prec x_{2}$ and $x_{1} \succ x_{2}$.

Let us introduce a notion of invariant bilinear map on a dendriform algebra. It is an antisymmetric map:

$$
\langle x, y\rangle=-\langle y, x\rangle
$$

such that

$$
\langle x \succ y, z\rangle=\langle y \prec z, x\rangle \text { and }\langle x \prec y, z\rangle=-\langle y \prec z, x\rangle-\langle y \succ z, x\rangle .
$$

As before, let us define a map $\tau_{1}$ as multiplication by -1 on $\operatorname{Dend}(1)$ and a map $\tau_{2}$ on $\operatorname{Dend}(2)$ by the following relation

$$
\langle E(x, y), z\rangle=\left\langle\tau_{2}(E)(y, z), x\right\rangle
$$

for each element $E$ in the base $Y^{2}$ of Dend(2), using the previous conditions on $\langle$,$\rangle .$

Theorem 4.1 There exists a unique collection $\tau$ of endomorphisms $\tau_{n}$ of the space $\operatorname{Dend}(n)$ extending $\tau_{1}$ and $\tau_{2}$ and endowing the operad Dend with a structure of non-symmetric anticyclic operad.

Proof Clearly $\tau_{1}$ is of order 2 and $\tau_{2}$ is of order 3 . As Dend is generated by Dend(2), the maps $\tau$ are unique if they exist. To check that $\tau_{n}$ can be defined for $n \geq 3$, it is enough to check that the notion of invariant form is compatible

with the three relations defining the operad Dend. Let us check that it works for the second compatibility condition:

$$
\begin{gathered}
\langle x \succ(y \prec z)-(x \succ y) \prec z, t\rangle \\
=\langle(y \prec z) \prec t, x\rangle+\langle z \prec t, x \succ y\rangle+\langle z \succ t, x \succ y\rangle \\
=\langle(y \prec z) \prec t, x\rangle-\langle x \succ y, z \prec t\rangle-\langle x \succ y, z \succ t\rangle \\
=\langle(y \prec z) \prec t, x\rangle-\langle y \prec(z \prec t)+y \prec(z \succ t), x\rangle=0,
\end{gathered}
$$


where one has used the antisymmetry and the invariance to obtain an expression with the $x$ variable alone on the right. The two remaining computations are just as simple and are left to the reader.

The matrix of $\tau$ in the base of trees certainly deserves more study. It seems to be related to the so-called Tamari lattices [8].

\section{$5 \quad$ Four operads}

Starting from this section, we consider operads in the usual sense, which means with actions of the symmetric groups. As explained in section 1, we can consider the two non-symmetric anticyclic operads just defined as anticyclic operads, still denoted Dias and Dend. We will show that some sub-operads and quotient operads of these inherit an anticyclic structure.

\subsection{The Perm operad}

The Perm operad, introduced in 2], is a quotient operad of the diassociative operad Dias. The space $\operatorname{Perm}(n)$ has dimension $n$ and the action of $\mathfrak{S}_{n}$ is the usual permutation representation.

The operad Perm is the quotient of Dias by the ideal generated by the element $x_{1} \dashv x_{2}-x_{2} \vdash x_{1}$. The image of the product $x_{1} \dashv x_{2}$ will be denoted $x_{1} x_{2}$.

The operad Perm is quadratic, generated by the binary product $x y$ (regular representation of $\mathfrak{S}_{2}$ ) and with relations

$$
(x y) z=x(y z)=x(z y) .
$$

Theorem 5.1 The operad Perm has a unique structure of anticyclic operad such that the quotient map from Dias is a morphism of anticyclic operads.

Proof One has to check that the defining ideal is stable by the action of $\mathfrak{S}_{n+1}$ on $\operatorname{Dias}(n)$. It is enough to check this in $\operatorname{Dias}(2)$, which contains the generators of the ideal, where it is immediate. Hence Perm is a quotient anticyclic operad of Dias. 
The resulting notion of invariant bilinear map is as follows. It is an antisymmetric map

$$
\langle x, y\rangle=-\langle y, x\rangle
$$

such that

$$
\langle x y, z\rangle=-\langle z y, x\rangle \text { and }\langle x y, z\rangle=\langle x z, y\rangle-\langle z x, y\rangle \text {. }
$$

Theorem 5.2 The action of $\mathfrak{S}_{n+1}$ on $\operatorname{Perm}(n)$ is isomorphic to the representation by reflections.

Proof Let us consider the action of $\mathfrak{S}_{n+1}$ by permutations on the module with base $\left(\varepsilon_{i}\right)_{i=1, \ldots, n+1}$. The reflection module is the submodule with base $b_{m}^{n}=\varepsilon_{m}-\varepsilon_{n+1}$ for $m=1, \ldots, n$. The action of the subgroup $\mathfrak{S}_{n}$ is by permutations of the vectors $b_{m}^{n}$ and the action of the cycle $\tau_{n}:(n+1, n, \ldots, 2,1)$ is given by

$$
\tau_{n}\left(b_{m}^{n}\right)= \begin{cases}-b_{n}^{n} & \text { if } m=1, \\ -b_{n}^{n}+e_{m-1}^{n} & \text { else. }\end{cases}
$$

On the other hand, the action of $\mathfrak{S}_{n}$ on the module Perm $(n)$ with base $\left(e_{m}^{n}\right)_{m=1, \ldots, n}$ is by permutation of the vectors $e_{m}^{n}$. This base of $\operatorname{Perm}(n)$ is the image of the base $e_{m}^{n} \otimes 1$ of the operad Dend. Hence the action of the cycle $\tau_{n}$ is induced by the action of the cycle $\tau_{n}$ in the base $e_{m}^{n}$ of the non-symmetric operad Dias which is given by Formula (24). This concludes the proof.

Corollary 5.3 The characteristic function of the anticyclic operad Perm is

$$
\mathbf{C h}(\operatorname{Perm})=\left(p_{1}-1\right) \exp \left(\sum_{k \geq 1} \frac{p_{k}}{k}\right)+1 .
$$

Proof This follows readily from the previous Theorem and the well-known fact that

$$
\operatorname{ch}(\mathrm{Comm})=\exp \left(\sum_{k \geq 1} \frac{p_{k}}{k}\right)-1
$$

\subsection{The Leibniz operad}

The Leibniz operad is a sub-operad of Dias, also introduced in [1]. It will be denoted by Leib. 
It is the sub-operad of Dias generated by the element $\left[x_{1}, x_{2}\right]=x_{1} \dashv x_{2}-x_{2} \vdash$ $x_{1}$. Beware that this bracket is not antisymmetric. Leibniz algebras are "noncommutative Lie algebras" in some sense.

The quadratic presentation of Leib is the following. It is generated by the binary product $[x, y]$ (regular module of $\mathfrak{S}_{2}$ ) modulo the relation

$$
[x,[y, z]]=[[x, y], z]-[[x, z], y] .
$$

It is known that the space $\operatorname{Leib}(n)$ is the regular representation of $\mathfrak{S}_{n}$, with base given by left-bracketed words in the variables $x_{1}, \ldots, x_{n}$.

Theorem 5.4 The operad Leib has a unique structure of anticyclic operad such that the inclusion map into Dias is a morphism of anticyclic operads.

Proof It is enough to check that Leib(2) is indeed a submodule of Dias(2) for the action of $\mathfrak{S}_{3}$. This is immediate and implies that $\operatorname{Leib}(n)$ is stable by the action of the symmetric group $\mathfrak{S}_{n+1}$ on $\operatorname{Dias}(n)$.

The resulting notion of invariant bilinear map is as follows. It is an antisymmetric map

$$
\langle x, y\rangle=-\langle y, x\rangle
$$

such that

$$
\langle[x, y], z\rangle=\langle[z, y], x\rangle \text { and }\langle[x, y], z\rangle=-\langle[z, x], y\rangle-\langle[x, z], y\rangle \text {. }
$$

Theorem 5.5 The action of $\mathfrak{S}_{n+1}$ on $\operatorname{Leib}(n)$ is isomorphic to the Lie module $\operatorname{Lie}(n+1)$.

Proof It was proved in [2] that the operad Leib is the Hadamard product of the operads Perm and Lie. As explained in Section 1, the Hadamard product of a cyclic operad and an anticyclic operad is an anticyclic operad. One can check that the anticyclic structure obtained in this way on Leib coincides with the one introduced above. Hence the action of $\mathfrak{S}_{n+1}$ on Leib $(n)$ is given by the tensor product of the $\mathfrak{S}_{n+1}$-modules $\operatorname{Lie}(n)$ and $\operatorname{Perm}(n)$. Then using Theorem [5.2 and [5. Corollary 6.8], this is known to be isomorphic to the module $\operatorname{Lie}(n+1)$. 


\subsection{The Pre-Lie operad}

The PreLie operad has been introduced in [3]. It is the sub-operad of the dendriform operad generated by the operation $x_{1} \curvearrowleft x_{2}=x_{2} \succ x_{1}-x_{1} \prec x_{2}$. The space PreLie $(n)$ has dimension $n^{n-1}$.

Let us recall the presentation of the operad PreLie. The product $x \curvearrowleft y$ is the regular module for $\mathfrak{S}_{2}$ and must satisfy the following relation:

$$
(x \curvearrowleft y) \curvearrowleft z-x \curvearrowleft(y \curvearrowleft z)=(x \curvearrowleft z) \curvearrowleft y-x \curvearrowleft(z \curvearrowleft y) .
$$

Theorem 5.6 The operad PreLie has a unique structure of anticyclic operad such that the inclusion map into Dend is a morphism of anticyclic operads.

Proof It is enough to check that PreLie(2) is indeed a submodule of Dend(2) for the $\mathfrak{S}_{3}$ action. This is immediate and implies that PreLie $(n)$ is stable by the action of the symmetric group $\mathfrak{S}_{n+1}$ on $\operatorname{Dend}(n)$.

The resulting notion of invariant bilinear map is as follows. It is an antisymmetric map

$$
\langle x, y\rangle=-\langle y, x\rangle
$$

such that

$$
\langle x \curvearrowleft y, z\rangle=-\langle x \curvearrowleft z, y\rangle \text { and }\langle x \curvearrowleft y, z\rangle=-\langle y \curvearrowleft z, x\rangle+\langle z \curvearrowleft y, x\rangle .
$$

Remark As the space $\operatorname{PreLie}(n)$ is isomorphic as a $\mathfrak{S}_{n}$-module to the space with a base indexed by labelled rooted trees on $n$ vertices, this implies the existence of a remarkable linear action of $\mathfrak{S}_{n+1}$ on this space.

Theorem 5.7 One has the following equality of symmetric functions:

$$
\operatorname{ch}(\text { PreLie })(1+\mathbf{C h}(\text { PreLie }))=p_{1}\left(1+\operatorname{ch}(\text { PreLie })+\operatorname{ch}(\text { PreLie })^{2}\right) .
$$

Proof One can check that PreLie is the Koszul dual anticyclic operad of Perm. Hence its characteristic function is obtained by a Legendre transform of the characteristic function of Perm.

But since Perm and PreLie are Koszul dual operads, it is known that

$$
\operatorname{ch}(\text { Perm }) \circ \Sigma \operatorname{ch}(\text { PreLie })=p_{1} \text {. }
$$

This can be used to replace the equation defining the Legendre transform of $\mathbf{C h}($ Perm $)$ by a relation no longer involving the plethysm. Applying the suspension $\Sigma$ to this relation leads to Formula (50). 
We propose here an explicit conjecture for the character of $\mathfrak{S}_{n+1}$ on $\operatorname{PreLie}(n)$ as a symmetric function.

Conjecture 5.8 The characteristic function $\mathbf{C h}$ (PreLie) of the anticyclic operad PreLie is

$$
\sum_{\lambda,|\lambda| \geq 1, \lambda_{1} \neq 1}\left(\lambda_{1}-1\right)^{\lambda_{1}-2} \prod_{k \geq 2}\left(\left(f_{k}(\lambda)-1\right)^{\lambda_{k}}-k \lambda_{k}\left(f_{k}(\lambda)-1\right)^{\lambda_{k}-1}\right) \frac{p_{\lambda}}{z_{\lambda}},
$$

where the sums runs over non-empty partitions $\lambda$ not having exactly one part of size $1, \lambda_{k}$ denotes the number of parts of size $k$ in the partition $\lambda$ and $f_{k}(\lambda)$ denotes the number of fixed points of the $k^{\text {th }}$ power of a permutation of cycle type $\lambda$. The notations $p_{\lambda}$ and $z_{\lambda}$ are classical for power sum symmetric functions and related constants.

It is easy to check that the restriction to $\mathfrak{S}_{n}$ gives back the known formula for the action on rooted trees which can be found in [10. It has been checked by computer up to $n=14$ that the expected characteristic function is indeed a positive linear combination of Schur functions and that Formula (50) holds.

\subsection{The Zinbiel operad}

The Zinbiel operad, denoted by Zinb, was introduced in 11]. Maybe it would be more appealing to call it the shuffle operad. It is the quotient operad of Dend by the ideal generated by the following element

$$
x_{1} \prec x_{2}-x_{2} \succ x_{1} \text {. }
$$

The image in Zinb of the product $x_{1} \prec x_{2}$ will be denoted $x_{1} x_{2}$.

The operad Zinb has a quadratic presentation. It is generated by the binary product $x y$ (regular representation of $\mathfrak{S}_{2}$ ) subject to the relation:

$$
(x y) z=x(y z)+x(z y) .
$$

The space $\operatorname{Zinb}(n)$ is isomorphic to the regular representation of $\mathfrak{S}_{n}$ and the composition of the operad can be described using shuffles of permutations.

Theorem 5.9 The operad Zinb has a unique structure of anticyclic operad such that the quotient map from Dend is a morphism of anticyclic operads.

Proof Once again, it follows already from the check done for the PreLie operad that the ideal defining Zinb is indeed stable by the action of the symmetric group $\mathfrak{S}_{n+1}$ on Dend $(n)$. Hence Zinb is a quotient anticyclic operad of Dend. 
The resulting notion of invariant bilinear map is as follows. It is an antisymmetric map

$$
\langle x, y\rangle=-\langle y, x\rangle
$$

such that

$$
\langle x y, z\rangle=\langle x z, y\rangle \text { and }\langle x y, z\rangle=-\langle y z, x\rangle-\langle z y, x\rangle \text {. }
$$

Theorem 5.10 The action of $\mathfrak{S}_{n+1}$ on $\operatorname{Zinb}(n)$ is isomorphic to the Lie module $\operatorname{Lie}(n+1)$.

Proof It is known that the operad Zinb is the Koszul dual of the operad Leib. One can check that the anticyclic structure of Leib is obtained from the anticyclic structure of Zinb by Koszul duality. Hence it follows that the characteristic of the anticyclic operad Zinb is related to the characteristic of the anticyclic operad Leib by a Legendre transform of symmetric functions. The characteristic of Leib is known by Theorem 5.5 to be

$$
F=\sum_{n \geq 2} \frac{1}{n} \sum_{d \mid n} \mu(d) p_{d}^{n / d} .
$$

One has to check that the Legendre transform of $F$ is

$$
G=\sum_{n \geq 2} \frac{1}{n} \sum_{d \mid n} \mu(d)(-1)^{n / d} p_{d}^{n / d} .
$$

The derivative of $G$ is

$$
\partial_{p_{1}} G=\frac{p_{1}}{1+p_{1}}
$$

and one has

$$
p_{1} \partial_{p_{1}} G=\frac{p_{1}^{2}}{1+p_{1}} .
$$

Let us compute $F \circ \partial_{p_{1}} G+G$. One finds

$$
\sum_{n \geq 2} \frac{1}{n} \sum_{d \mid n} \mu(d)\left(\frac{1}{\left(1+p_{d}\right)^{n / d}}+(-1)^{n / d}\right) p_{d}^{n / d} .
$$

Now fix $d \geq 2$ and look only at the extracted series in $p_{d}$ :

$$
\frac{\mu(d)}{d} \sum_{k \geq 1} \frac{1}{k}\left(\frac{1}{\left(1+p_{d}\right)^{k}}+(-1)^{k}\right) p_{d}^{k}
$$


This is easily expressed using logarithms and seen to vanish. Hence the expression $F \circ \partial_{p_{1}} G+G$ is given by

$$
\sum_{n \geq 2} \frac{1}{n}\left(\frac{1}{\left(1+p_{1}\right)^{n}}+(-1)^{n}\right) p_{1}^{n}=\frac{p_{1}^{2}}{1+p_{1}}
$$

as expected.

Let us remark that exactly the same computation with the Möbius function $\mu$ replaced by the Euler totient function $\varphi$ corresponds to self-duality of the characteristic function for the cyclic Koszul operad Assoc.

\section{Characters of Dend and Dias}

Theorem 6.1 The characteristic function of the anticyclic operad Dias is

$$
\mathbf{C h}(\text { Dias })=\sum_{n \geq 2}\left(p_{1}^{n}-\frac{1}{n} \sum_{d \mid n} \varphi(d) p_{d}^{n / d}\right) .
$$

Proof It is easy to check that the anticyclic operad Dias is obtained as the Hadamard product of the anticyclic operad Perm by the cyclic operad Assoc.

It is known that the characteristic function of the cyclic operad Assoc is

$$
\sum_{n \geq 2} \frac{1}{n} \sum_{d \mid n} \varphi(d) p_{d}^{n / d}
$$

where $\varphi$ is the Euler totient function.

Using Theorem [5.2, one knows that the tensor product by the $\mathfrak{S}_{n+1}$ module $\operatorname{Perm}(n)$ is given by the operator $-\mathrm{Id}+\partial_{p_{1}}$. Then a simple computation proves the Theorem.

One can check that the anticyclic structure of Dend is the one obtained from the anticyclic structure of Dias by Koszul duality. Hence, by Legendre inversion, one gets that the characteristic function of Dend is related to the Legendre transform of the characteristic function of Dias.

Theorem 6.2 The characteristic function of the anticyclic operad Dend is

$$
\mathbf{C h}(\text { Dend })=1-p_{1}-\sqrt{1-4 p_{1}}-\sum_{n \geq 1}\left(\frac{1}{2 n} \sum_{d \mid n} \varphi(d)\left(\begin{array}{c}
2 n / d \\
n / d
\end{array}\right) p_{d}^{n / d}\right) \text {. }
$$


Proof Let us check that the Legendre transform gives this result. Let $F$ be the characteristic function (64) of Dias and let $G$ be

$$
1+p_{1}-\sqrt{1+4 p_{1}}-\sum_{n \geq 1} \frac{1}{2 n} \sum_{d \mid n} \varphi(d)\left(\begin{array}{c}
2 n / d \\
n / d
\end{array}\right)\left(-p_{d}\right)^{n / d} .
$$

One has to check that $F \circ \partial_{p_{1}} G+G=p_{1} \partial_{p_{1}} G$.

Then one has

$$
\partial_{p_{1}} G=\frac{1+2 p_{1}-\sqrt{1+4 p_{1}}}{2 p_{1}}
$$

and

$$
p_{1} \partial_{p_{1}} G=\frac{1+2 p_{1}-\sqrt{1+4 p_{1}}}{2} .
$$

Let us compute $p_{1} \partial_{p_{1}} G-F \circ \partial_{p_{1}} G$. One finds

$$
\begin{gathered}
\frac{1+2 p_{1}-\sqrt{1+4 p_{1}}}{2}-\left(\frac{1+2 p_{1}-\sqrt{1+4 p_{1}}}{2 p_{1}}\right)^{2} \frac{1}{1-\left(\frac{1+2 p_{1}-\sqrt{1+4 p_{1}}}{2 p_{1}}\right)} \\
+\sum_{n \geq 2} \frac{1}{n} \sum_{d \mid n} \varphi(d)\left(\frac{1+2 p_{d}-\sqrt{1+4 p_{d}}}{2 p_{d}}\right)^{n / d} .
\end{gathered}
$$

Let us split this sum into

$$
\frac{1+2 p_{1}-\sqrt{1+4 p_{1}}}{2}-\frac{\left(1+2 p_{1}-\sqrt{1+4 p_{1}}\right)^{2}}{2 p_{1}\left(\sqrt{1+4 p_{1}}-1\right)}-\frac{1+2 p_{1}-\sqrt{1+4 p_{1}}}{2 p_{1}}
$$

and

$$
\sum_{n \geq 1} \frac{1}{n} \sum_{d \mid n} \varphi(d)\left(\frac{1+2 p_{d}-\sqrt{1+4 p_{d}}}{2 p_{d}}\right)^{n / d} .
$$

Let us compute these separately. The first part is easily seen to be

$$
1+p_{1}-\sqrt{1+4 p_{1}} \text {. }
$$

The second part becomes

$$
\sum_{d \geq 1} \frac{\varphi(d)}{d} \sum_{k \geq 1} \frac{1}{k}\left(\frac{1+2 p_{d}-\sqrt{1+4 p_{d}}}{2 p_{d}}\right)^{k},
$$

which is

$$
\sum_{d \geq 1} \frac{\varphi(d)}{d} \log \left(\frac{1+\sqrt{1-4 p_{d}}}{2}\right)
$$


Using then the Taylor expansion

$$
-\log \left(\frac{1+\sqrt{1-4 u}}{2}\right)=\sum_{n \geq 1} \frac{1}{2 n}\left(\begin{array}{c}
2 n \\
n
\end{array}\right) u^{n},
$$

one gets

$$
-\sum_{d \geq 1} \frac{\varphi(d)}{d} \sum_{k \geq 1} \frac{1}{2 k}\left(\begin{array}{c}
2 k \\
k
\end{array}\right)\left(-p_{d}\right)^{k}
$$

which becomes

$$
-\sum_{n \geq 1} \frac{1}{2 n} \sum_{d \mid n} \varphi(d)\left(\begin{array}{c}
2 n / d \\
n / d
\end{array}\right)\left(-p_{d}\right)^{n / d} .
$$

Summing both parts gives, as expected, that $p_{1} \partial_{p_{1}} G-F \circ \partial_{p_{1}} G=G$.

\section{References}

[1] Marcelo Aguiar, Pre-Poisson algebras, Lett. Math. Phys. 54 (2000) 263-277 MathReview

[2] Frédéric Chapoton, Un endofoncteur de la catégorie des opérades, from: "Dialgebras and related operads", Lecture Notes in Math. 1763, Springer, Berlin (2001) 105-110 MathReview

[3] Frédéric Chapoton, Muriel Livernet, Pre-Lie algebras and the rooted trees operad, Internat. Math. Res. Notices (2001) 395-408 MathReview

[4] James Conant, Karen Vogtmann, On a theorem of Kontsevich, Algebr. Geom. Topol. 3 (2003) 1167-1224 MathReview

[5] E Getzler, MM Kapranov, Cyclic operads and cyclic homology, from: "Geometry, topology, \& physics", Conf. Proc. Lecture Notes Geom. Topology, IV, Internat. Press, Cambridge, MA (1995) 167-201 MathReview

[6] E Getzler, M M Kapranov, Modular operads, Compositio Math. 110 (1998) 65-126 MathReview

[7] Victor Ginzburg, Mikhail Kapranov, Koszul duality for operads, Duke Math. J. 76 (1994) 203-272 MathReview

[8] Samuel Huang, Dov Tamari, Problems of associativity: A simple proof for the lattice property of systems ordered by a semi-associative law, J. Combinatorial Theory Ser. A 13 (1972) 7-13 MathReview

[9] Maxim Kontsevich, Formal (non)commutative symplectic geometry, from: "The Gelfand Mathematical Seminars, 1990-1992", Birkhäuser Boston, Boston, MA (1993) 173-187 MathReview 
[10] Gilbert Labelle, Some new computational methods in the theory of species, from: "Combinatoire énumérative", Lecture Notes in Math. 1234, Springer, Berlin (1986) 192-209 MathReview

[11] Jean-Louis Loday, Dialgebras, from: "Dialgebras and related operads", Lecture Notes in Math. 1763, Springer, Berlin (2001) 7-66 MathReview

[12] Martin Markl, Cyclic operads and homology of graph complexes, Rend. Circ. Mat. Palermo (2) Suppl. (1999) 161-170, the 18th Winter School "Geometry and Physics" (Srní, 1998) MathReview

Institut Girard Desargues, Université Claude Bernard (Lyon 1)

Bâtiment Braconnier, 21 Avenue Claude Bernard

F-69622 Villeurbanne Cedex, France

Email: chapoton@igd.univ-lyon1.fr

Received: 6 December 2004 